\title{
Nonlinear evolution of the surface morphology under shadowing
}

\author{
P. Manz ${ }^{1}$, N. Fedorczak ${ }^{2}$, T. Dittmar ${ }^{3}$, T. Baloniak ${ }^{4}$, A. von Keudell ${ }^{4}$ \\ 1 Max-Planck-Institut für Plasmaphysik, Association Euratom-IPP, 85748 Garching, Germany \\ 2 Commissariat à l Energie Atomique et aux Energies Alternatives, \\ Institut de Recherche pour la Fusion Magneétique Controlée, F-13108 Saint-Paul-Lez-Durance, France \\ ${ }^{3}$ Center for Energy Research, University of California at San Diego, San Diego, California 92093, USA \\ 4 Research Department Plasmas With Complex Interactions, \\ Ruhr-Universität Bochum, 44780 Bochum, Germany
}

(Dated: March 6, 2013)

\begin{abstract}
Fluorocarbon thin-film deposition is studied, which shows an anomalous high dynamic growth exponent and therefore does not fit in any universal class of fractal surface growth models. A detailed analysis of the nonlinear behavior of the surface morphology evolution is carried out, quantifying several features of the shadowing instability. A synergy effect with the Kardar-Parisi-Zhang nonlinearity, which couple the large scales induced by shadowing with intermediate scales, may explain the anomalous high growth exponent.
\end{abstract}

PACS numbers:

\section{INTRODUCTION}

Thin film surface morphology controls many important physical and chemical properties of the films. It is therefore of great interest to understand and control the evolution of the surface morphology during thin film growth. For example very rough surfaces have a low wettability and can result in so-called super-hydrophobic films with applications in waterproofing of textiles or biocompatible layers in medical devices. Rough surfaces are characterized by a complex fractal-like surface morphology. As fractals results from the nonlinear dynamic of the system, nonlinear processes as spontaneous pattern formation are strongly linked with rough surfaces. Thus, spontaneous pattern formation during surface processing is a way to fabricate nanoscale textured (rough) materials. The kinetic roughening of many surfaces follows rather simple scaling laws [1]. Within this scaling theory, the surface roughness can be represented by the root-mean-square $w$ of fluctuations of the surface height $h(\mathbf{r}, t)$. At early times during deposition, the surface roughness should scale with the time $w \sim t^{\beta}$, where $\beta$ is the growth exponent. The growth saturates after some time $t_{x}$ and the surface roughness should scale with the system size $w \sim L^{\alpha}$, where $\alpha$ is called the roughness exponent. These exponents allow to classify different universality classes, providing informations about the underlying equations determining the deposition process.

Continuum growth equations are valid in the small slope approximation $|\nabla h| \ll 1$ but with increasing system size, the slopes increase and the saturation can be dominated by nonlinear processes. Therefore, several universal classes include nonlinear terms and also other processes governing the film growth as redeposition [2] or shadowing [7] are nonlinear. Thus, there is a necessity to study the nonlinear behavior during deposition. Previous experimental investigations of the surface roughening have been restricted to the determination of the scaling exponents $\alpha$ and $\beta[4-6]$. More detailed analy- ses are in particular necessary, if the experimental scaling exponents do not fit to any universal class. Such an anomalous scaling have been reported from fluorocarbon thin films deposition [4], where shadowing has been expected to be responsible for the large $\beta$. This study presents a detailed nonlinear analysis of the surface morphology of fluorocarbon thin films deposition. To gain inside into the shadowing mechanism the analysis of experimental data is accompanied by a simple Monte Carlo simulation of pure shadowing to characterize, quantify and distinguish the effects of shadowing against other possible roughening mechanisms. It is found that the experimentally observed surface morphology shows all features of shadowing. The development of large scale structures on the surface is due to shadowing. Although strongly present shadowing alone cannot account for the observed large $\beta$. The Kardar-Parisi-Zhang (KPZ) nonlinearity redistributes the surface height perturbations among the scales and transfers the perturbations from the large-scale structures induced by the shadowing to smaller scales. This synergy effect between these two nonlinearities may be indeed responsible for the anomalous scaling reported in Ref. [4].

\section{EXPERIMENTAL SETUP AND PREVIOUS RESULTS}

Fluorocarbon films were deposited on polished $\mathrm{Si}$ wafers on a grounded electrode in a capacitively coupled plasma reactor driven by an $\mathrm{RF}$ generator at $13.56 \mathrm{MHz}$. The distance between the grounded and driven electrode was $39 \mathrm{~mm}$ and the total electrode surface area was 43 $\mathrm{cm}^{2}$. As feedstock $\mathrm{c}-\mathrm{C}_{4}-\mathrm{F}_{8}$ was used. The discharge was operated at $100 \mathrm{~Pa}$ and a power of $60 \mathrm{~W}$ was applied. The gas flow was $17 \mathrm{sccm}$. More details on the experimental apparatus can be found in [4]. The surface morphology of the samples was studied ex situ by atomic force microscopy (AFM). For AFM measurements no intrinsic 
a)

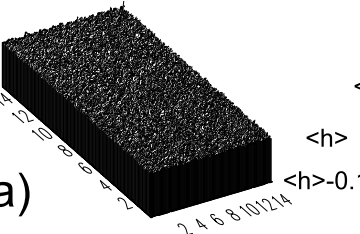

e)

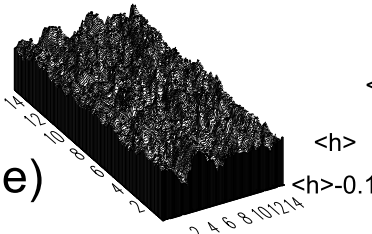

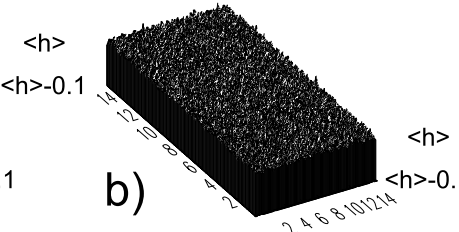

b)

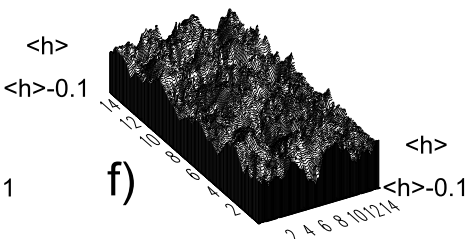

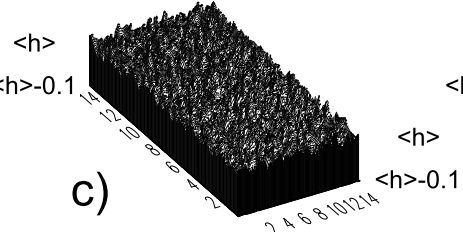

c)

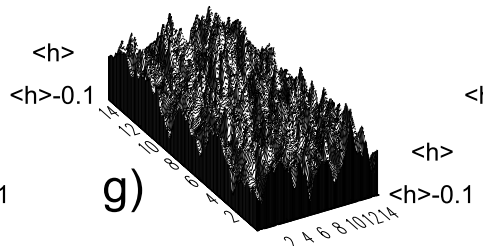

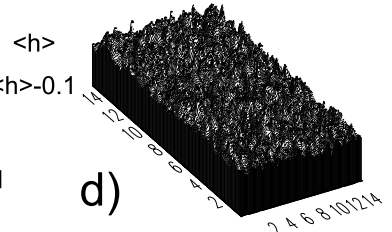

d)

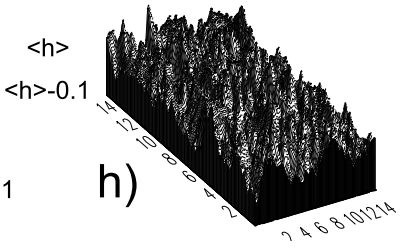

FIG. 1: AFM images $(15 \mu m \times 15 \mu m)$ at different times and film thickness (a) $\langle h\rangle=0.050$, (b) 0.075, (c) $0.100,(d) 0.125,(e)$ 0.150 , (f) 0.200, (g) 0.250 and (h) $0.300 \mu \mathrm{m}$ respectively.

nonlinearities in the data acquisition are known. Images of $15 \mu \mathrm{m} \times 15 \mu \mathrm{m}$ were taken at a resolution of $256 \times 256$ points as shown in [4]. A dynamic scaling exponent of $\beta=1.35 \pm 0.08$ has been found. Such a large growth exponent does not fit to any growth model found in [1]. The largest dynamical scaling exponents (up to one) are predicted by shadowing [11]. Therefore it can be expected that shadowing play an important role during the deposition process. As shown in Fig. 1a and b, the surface shows a grassy topology at early times, which is indicative for geometrical shadowing effects $[1,3]$. However, shortly after that, cusps develop. These cusps are larger structures taking over the smaller ones (Fig. 1c and d) as expected for shadowing where taller surface features block incoming flux from reaching lower lying areas of the surface $[1,3]$. However as $\beta>1$ another roughening mechanism which is synchronized to shadowing must be present or the nonlinearity redistributes the spectral power already deposited on the surface and additional roughens the surface by means of this process. The static scaling exponent $\alpha$ increases during the first minutes of deposition from $0.4((\mathrm{a})$ and $(\mathrm{b}))$ to $0.6(\mathrm{~d})$ and then saturates at a value of $\alpha=0.67 \pm 0.02$. In the case of oblique incidence during sputter deposition a value of $\alpha=2 / 3$ is predicted by nonlinear growth including surface diffusion. In this particular case a $\beta$ of $1 / 5$ is predicted, which is not observed in the experiment. Anyhow, in the experiments reported here the particles do not arrive at the surface under a single oblique angle. A value of $\alpha=1$ indicate linear growth, a value of $\alpha=1 / 2$ can correspond to nonlinear growth with relaxation due to surface tension [1]. Again, none of the universal classes reported in [1] is consistent with the experiment, but the observed static scaling exponent $\alpha \approx 2 / 3$ points to a further nonlinear mechanism during the deposition.

\section{SHADOWING}

The primary nonlocal effect is the shadowing effect [3, 7-11], where taller surface features block incoming flux from reaching lower lying areas of the surface. The shadowing effect is active because the incoming flux has an angular distribution. This allows taller surface features to grow at the expense of shorter ones, leading to a competition between different surface features for particle flux. This competition leads to a grassy morphology and ultimately to a mounded surface as shorter surface features receive little or no particle flux and are suppressed. Shadowing is an inherently nonlocal process because the shadowing of a surface feature depends on the heights of all other surface features, not just close ones.

A model including both non-linear shadowing and diffusion effects has been initially developed by Bales and Zangwill [7] and by Karunasiri et al [8]. The roughening of a surface profile is given by the evolution of the surface height $h(x, t)$

$$
\frac{\partial h}{\partial t}=R \Omega \sqrt{1+(\nabla h)^{2}}+\nu \nabla^{2} h+\eta
$$

The stochastic arrival of the particles is modeled by white noise $\eta$. Relaxation is provided by the surface tension $\nu$. The deposition growth $R$ is multiplied with the exposure or solid angle $\Omega(x, h)$. The exposure angle gives a measure of the open sky that one can see from the position $x$. The factor $\sqrt{1+(\nabla h)^{2}}$ in Eq. (1) implies that the growth takes place normal to the surface [9]. Both the exposure angle and the normal growth are strongly nonlinear functions of the surface height and no analytical expression is known in Fourier wavenumber space.

The Fourier representation of Eq. (1) taking $\Psi=$ 
coherence

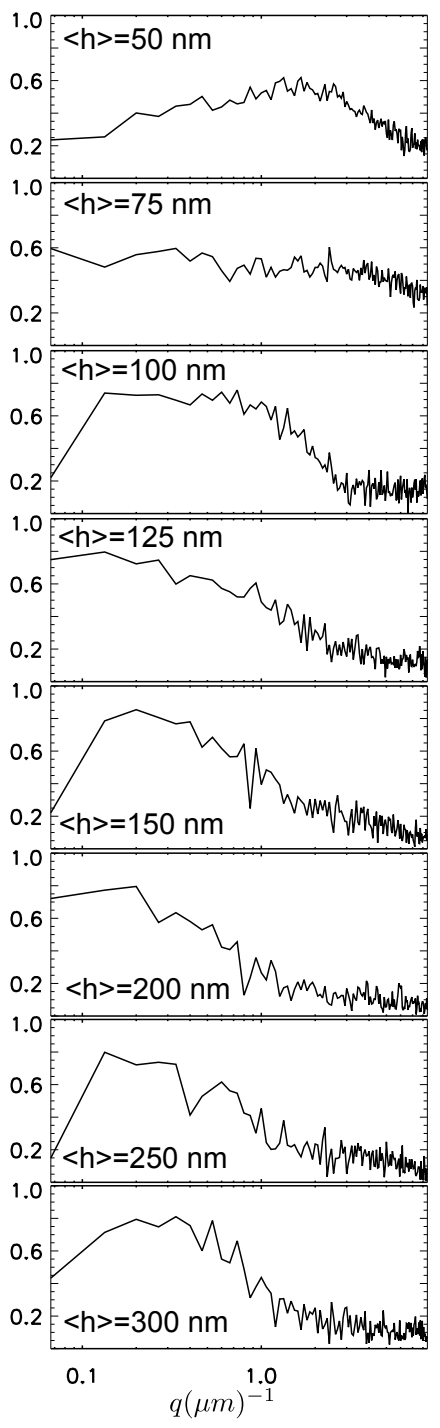

phase $/ \pi$

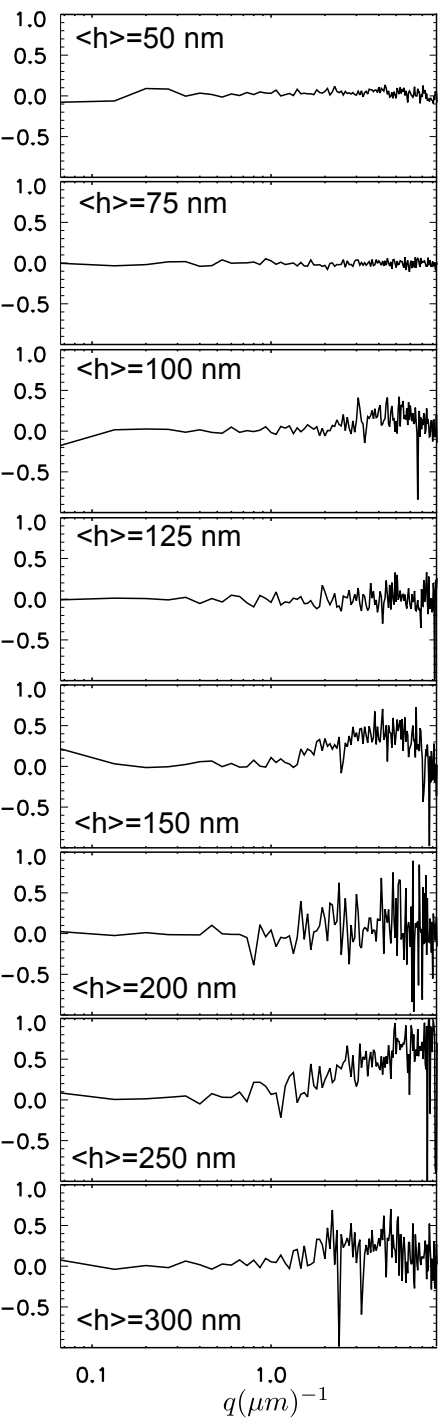

FIG. 2: Cross-coherence and -phase between surface height and exposure angle perturbations during fluorocarbon thinfilm deposition.

$$
\sqrt{1+(\nabla h)^{2}} \text { is }
$$

$$
\begin{aligned}
& \frac{\partial}{\partial t} \sum_{q_{1}} h\left(q_{1}, t\right) e^{i q_{1} x}= \\
& R \sum_{q_{2}} \Omega\left(q_{2}, t\right) e^{i q_{2} x} \sum_{q_{1}} \Psi\left(q_{1}, t\right)^{i q_{1} x} \\
& -\sum_{q_{1}} \nu q_{1}^{2} h\left(q_{1}, t\right) e^{i q_{1} x} .
\end{aligned}
$$

After multiplying both sides of this equation by $e^{-i q x}$ and integrating over $x$ the delta function condition for the shadowing term includes now three waves

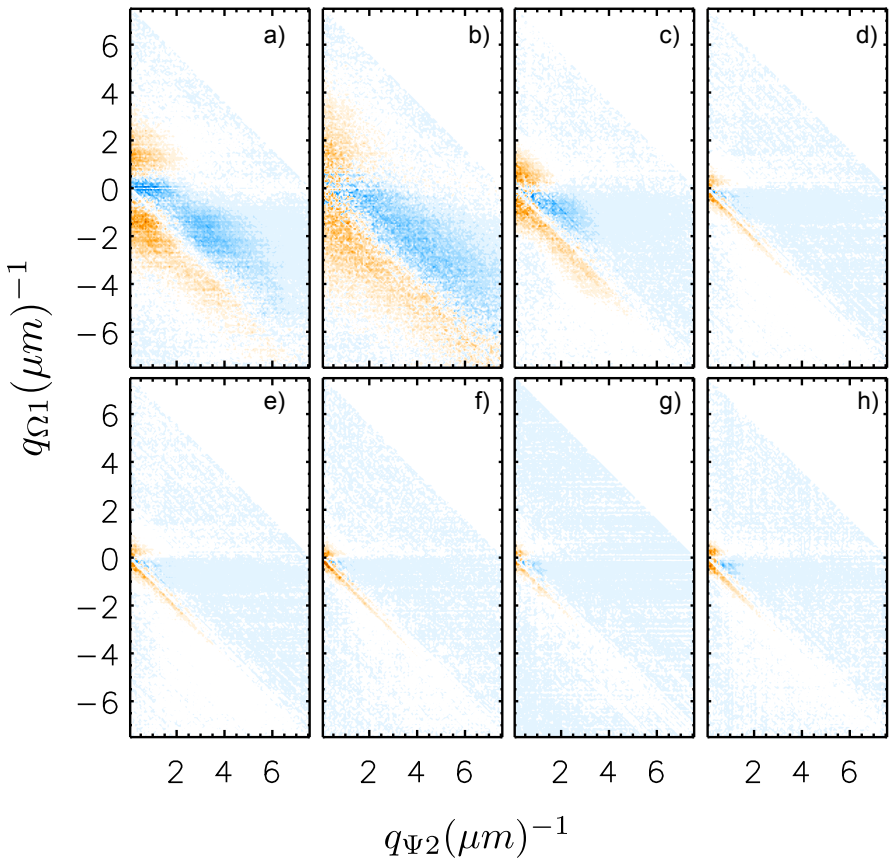

FIG. 3: Crossbispectrum between surface height, exposure angle and normal surface growth fluctuations during fluorocarbon thin-film deposition at different times and film thickness (a) $\langle h\rangle=0.050$, (b) 0.075, (c) 0.100, (d) 0.125, (e) 0.150, (f) $0.200,(\mathrm{~g}) 0.250$ and $(\mathrm{h}) 0.300 \mu \mathrm{m}$ respectively. The crossbispectrum is shown normalized to its maximum absolute value. Positive (negative) values are depicted in orange (blue). Informations on the impact of its amplitude can be found in Fig. 4.

$\int d x e^{i\left(q_{1}+q_{2}-q\right) x}=\delta\left(q_{1}+q_{2}-q\right)$. This results in

$$
\frac{\partial}{\partial t} h(q, t)=R \sum_{q=q_{1}+q_{2}} \Omega\left(q_{2}, t\right) \Psi\left(q_{1}, t\right)-\nu q^{2} h(q, t),
$$

or equivalent for the spectral density

$$
\begin{aligned}
\frac{1}{2} \frac{\partial}{\partial t} h^{2}(q, t)= & \operatorname{Re}\left(R \sum_{q=q_{1}+q_{2}} \Omega\left(q_{2}, t\right) \Psi\left(q_{1}, t\right) h^{*}(q, t)\right) \\
& -\nu q^{2} h^{2}(q, t)
\end{aligned}
$$

where the asterisk denotes the complex conjugate. Therefore in the large slope limit the exposure angle is nonlinearly interacting with the surface height perturbations and the normal growth fluctuations $\Psi$.

\section{A. Quasi-linear shadowing}

For small slopes $(\Psi \approx 1)$ and due to the strong contribution of the $q=0$ mode $\left(\Psi(q=0)=\sqrt{1+(q h)^{2}}=1\right)$ in general shadowing is in first order determined by the exposure angle. As this dependence is linear on the exposure angle, but the exposure angle depends on the surface height perturbations in a nonlinear nonlocal way, it 


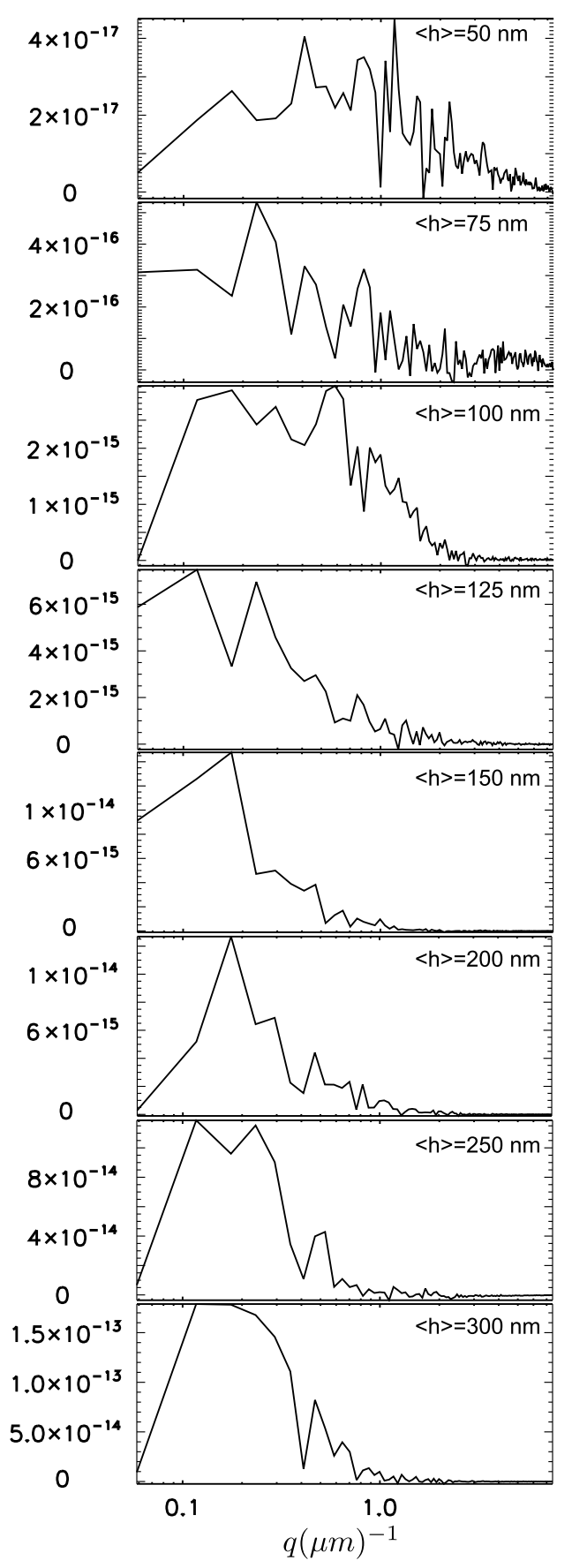

FIG. 4: Integrated crossbispectrum between surface height, exposure angle and normal surface growth fluctuations during fluorocarbon thin-film deposition.

is called quasi-linear here. Therefore in first order shadowing is given by the averaged cross-power spectrum as given by

$$
H_{h, \Omega}(q)=\left\langle h^{*}(q) \Omega(q)\right\rangle,
$$

where $h(q)$ and $\Omega(q)$ are the Fourier transforms in wavenumber. As a large number of samples for each condition for experimental data is not available, it seems

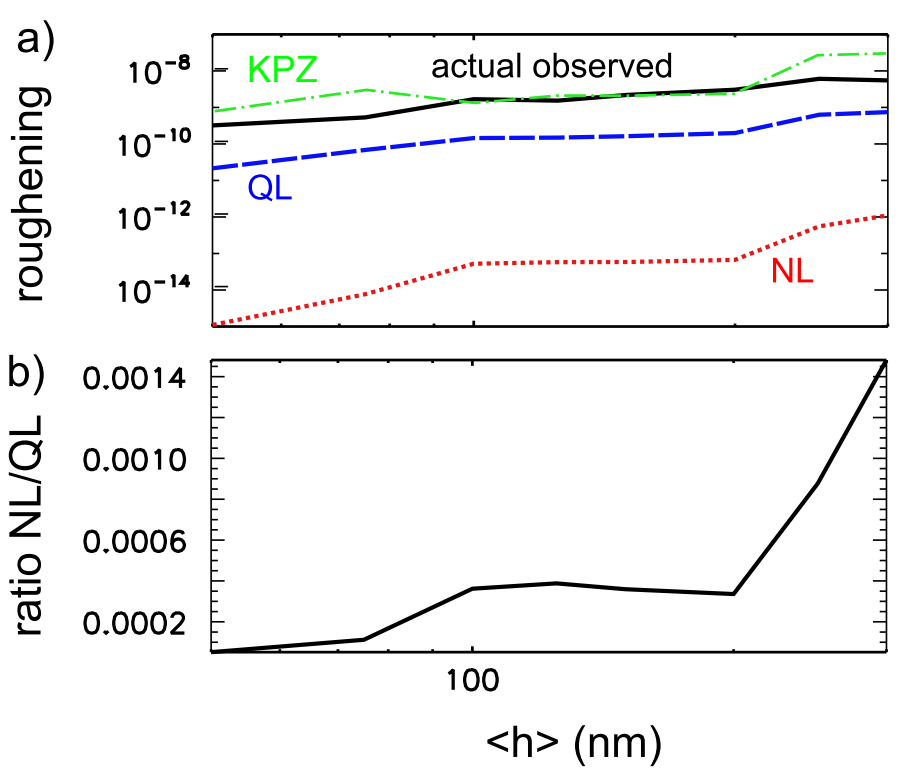

FIG. 5: (a) Comparison of the measured roughening (black, solid) with the roughening due to quasi-linear (QL, blue, dashed) and nonlinear shadowing (NL, red, dotted) and KPZ nonlinearity (KPZ, green, dash-dotted). (b) The ratio between nonlinear and quasi-linear roughening.

appropriate to carry out the the analysis in one dimension only, taking the second dimension as the statistical ensemble taking 128 sub-series of 128 points for ensemble averaging $\langle\cdot\rangle$. As a complex quantity, the cross-power spectrum can be written as $H_{h, \Omega}(q)=\left|H_{h, \Omega}(q)\right| e^{i \varphi_{h, \Omega}(q)}$, where $\varphi_{h, \Omega}(q)$ is the average cross-phase. The coherence, defined by

$$
\gamma_{h, \Omega}(q)=\frac{\left|\left\langle h^{*}(q) \Omega(q)\right\rangle\right|}{\sqrt{\langle|h(q)|\rangle^{2}\langle|\Omega(q)|\rangle^{2}}},
$$

measures, how phase locked modes are with values in $[0,1]$. Figure 2 shows the cross-coherence and the crossphase between the surface height and the exposure fluctuations. If a significant coherence is observed, the crossphase is close to zero. Therefore quasi-linear shadowing increases the spectral power and the surface roughness. With deposition time the coherence increases and shifts to larger structures (lower wave numbers). Therefore larger and larger structures are getting unstable to quasi-linear shadowing, where the smaller structures do only receive a marginal part of the particle flux. We observe that for these smaller structures the cross-phase between $h$ and $\Omega$ is getting more and more irregular as the deposition proceeds.

\section{B. Nonlinear shadowing}

Investigating scaling laws power spectral densities (PSD) are often used in surface topology analysis. How- 
ever, the PSD does not distinguish between independently excited and nonlinearly coupled waves. A measure of the quadratic coupling between two waves with wavelength $q_{1}$ and $q_{2}$ with a third one $q=q_{1}+q_{2}$ is given by the cross-bispectrum [12]

$$
B\left(q_{1}, q_{2}\right)=\left\langle\Omega\left(q_{1}\right) \Psi\left(q_{2}\right) h^{*}\left(q=q_{1}+q_{2}\right)\right\rangle .
$$

If these three waves are spontaneously, independently excited, their phases are also statistically independent and the bispectrum vanishes. On the other hand, a nonlinear coupling between the waves $q, q_{1}$ and $q_{2}$ is locking their phases with each other. As a result, the bispectrum takes a finite value. To investigate the fully nonlinear behavior the cross-bispectra $\left\langle\Omega\left(q_{1}\right) \Psi\left(q_{2}\right) h^{*}(q)\right\rangle$ have been calculated as shown in Fig. 3. For convenience the wave numbers in Fig. 3 are labeled with the quantity, which has been Fourier transformed $\left(q_{1} \equiv q_{\Omega 1}, q_{2} \equiv q_{\Psi 2}\right)$. All cross-bispectra show the same basic features. The region $q_{1}<0$ and $q_{1}+q_{2}>0$ is dominated by negative coupling $\left\langle\Omega\left(q_{1}\right) \Psi\left(q_{2}\right) h^{*}(q)\right\rangle<0$. For every surface height perturbation $h(q)$ an associated perturbation in the exposure angle $\Omega\left(q_{1}=q\right)$ can be found. As $\Psi \sim|\nabla h|$ the normal growth spectra $\Psi\left(q_{2}=2 q\right)$ contains the second harmonics of $h(q)$. The three-wave coupling condition is fulfilled. This damping caused by the coupling with the second harmonics reflects the smoothing of the surface due to the reduction of the slopes of the surface. The strongest nonlinear drive is found at $q_{1}<0, q_{2}>0$ and $q=q_{1}+q_{2}<0$ close to the line $q_{2}=-q_{1}$. This can result from the coupling $\Psi\left(q_{1}-q\right) \Omega^{*}\left(q_{1}\right) h^{*}(-q)$, where the modulation of the normal surface growth $\Psi\left(q_{1}-q\right)$ is used as a sideband to increase the spectral power in largescale features $h(q)$. It is found that two nonlinear mechanisms are active during the deposition, damping due to wavenumber doubling of the normal surface growth and growth due to its modulation by large-scale structures.

To estimate the impact of nonlinear shadowing the integrated cross-bispectra $\sum_{q=q_{1}+q_{2},-q=q_{1}+q_{2}}\left\langle\Omega\left(q_{1}\right) \Psi\left(q_{2}\right) h^{*}(q)\right\rangle$ are presented in Fig. 4. The cross-bispectra are integrated over both propagation directions $q$ and $-q$. It shows a similar behavior as the cross-coherence in Fig. 2. The integrated cross-bispectra are positive and therefore contribute to a roughening of the surface. With increasing deposition time the amplitude in the integrated cross-bispectra increases. The spectral power concentrates at large structures, which is also consistent with the shadowing instability. The impact of this nonlinear roughening will be investigated next.

The film thickness increases linearly in time $\langle h\rangle=R t$, where $R$ is the deposition growth rate. To compare the results presented here against previous work we use $\partial / \partial t=R \partial / \partial\langle h\rangle$. The evolution of the mean free spectral power $\left\langle h^{2}\right\rangle$ is related to the evolution of the roughness $w$ by $\frac{\partial\left\langle h^{2}\right\rangle}{\partial\langle h\rangle}=2 w \frac{\partial w}{\partial\langle h\rangle}$. Since the roughness develops according to a power law $w=w_{0}\langle h\rangle^{\beta}$ with growth exponent $\beta$ the time evolution is given by $\frac{\partial w}{\partial\langle h\rangle}=\beta w /\langle h\rangle$ which results in

$$
\frac{\partial\left\langle h^{2}\right\rangle}{\partial\langle h\rangle}=2 \beta \frac{w^{2}}{\langle h\rangle} .
$$

The actual observed roughening is compared to the quasi-linear (QL) and nonlinear (NL) roughening in Fig. 5. The quasi-linear shadowing is given by $\int_{q} \sqrt{\Omega^{2}(q)} \sqrt{h^{2}(q)} \cos (\Omega, h)$ where $\cos (\Omega, h)$ is the crossphase between surface height and exposure angle perturbations as shown on the right hand sight of Fig. 2. To be a possible candidate to explain the actual observed roughening it must be larger than that together with the damping which is not considered here. The nonlinear roughening given by $\int_{q} \sum_{q=q_{1}+q_{2},-q=q_{1}+q_{2}}\left\langle\Omega\left(q_{1}\right) \Psi\left(q_{2}\right) h^{*}(q)\right\rangle$ is also shown in Fig. 5. The observed anomalous dynamical scaling exponent $\beta=1.35$ of the experimental data cannot be explained by shadowing alone. As shown in Fig. 5a the roughening due to QL shadowing is about one order of magnitude below the actual observed one and the NL shadowing is even up to three orders of magnitude below that. For the experimental data NL shadowing is negligible (Fig. 5b). Therefore, the observed dynamic scaling coefficient $\beta$ can only result from another linear process, which should be cooperative to QL shadowing or from nonlinear redistribution of the spectral power by another nonlinearity as the KPZ-nonlinearity [13] for example as discussed in section IV.

\section{Monte Carlo Simulations}

The non-linearity of the shadowing effects can be easily tested on simple Monte Carlo simulations of the growth of a periodic one dimensional surface under incoming particle flux. An illustration is shown in Fig. 6. The method consists in sticking identically shaped incident particles on implementation sides at the boundary layer of the growing surface. All implementation sides are located at the nods of a uniform 2D mesh. Effects of binding energy and incident energy distribution are minimized to a simple form. A first layer of atoms is initiated with a required horizontal spectrum (red layer at the bottom of Fig. 6). A succession of particles is then sent from the top of the domain toward the surface, at a random discrete horizontal location with a random direction. At the discrete location where the particle trajectory hits the surface, the particle sticks with $99 \%$ of probability. If the receiving particle already sticked to the surface is only bounded to one other particle, within $1 \%$ probability both incident and receiving particles are remitted from that location, with uniform random directions and half of the initial probability to erode again. Results shown in Fig. 6 correspond to a periodic domain of 1024 sites, with about $10^{4}$ particles deposited above the initial surface. The high sticking and low erosion probability is responsible for a highly porous structure, because cascade processes are rare, and shadowing is clearly manifested. At each horizontal location $x$, the vertical position of the 
highest particle defines the surface height $h(x)$ at that position, as illustrated by the red curve at the top of the surface in Fig. 6. To perform statistical analyses on the surface growth dynamics, a series of 256 simulations has been performed with identical power spectrum for the initial surface roughness.

Surfaces obtained with this Monte Carlo approach give a roughness exponent of $\beta=0.95$, which value does not depend so much about the sticking coefficient. Indeed, for higher probability of surface erosion redeposition, the grass like structure as evident on Fig. 6 will exhibit a lower internal porosity, but the overall surface will keep a grassy like organization, due to each structure shadowing its neighboring ones. Comparing the experimental results with the those of the simulation, the qualitative agreement of the cross-coherence and cross-phase between $\Omega$ and $\Psi$ (Figs. 2 and 7 ), the cross-bicoherence between $\Omega$, $\Psi$ and $h$ (Figs. 3 and 8) and the integrated one (Figs. 4 and 9 ) is remarkable. It can be concluded that shadowing is strongly present during the deposition process.

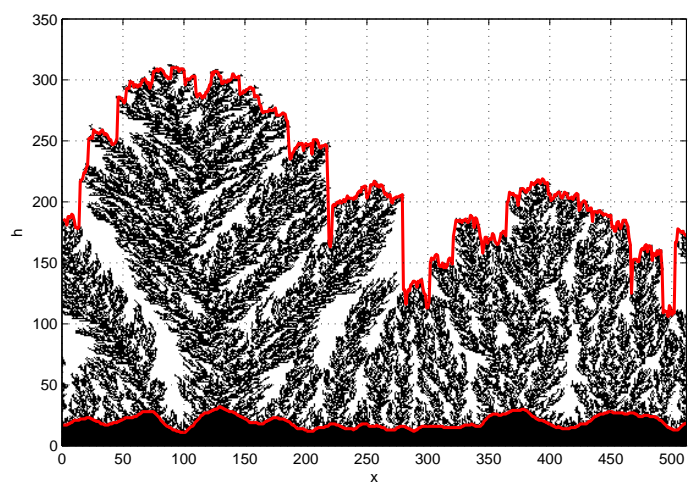

FIG. 6: Zoom on a grown surface under incident bombardment of highly sticking and low sputtering yield particles (in black). Implementation sites are discrete. The surface function $h(x)$ is locally defined by the position of the highest particle, as illustrated by the two red curves. The bottom one is the initial surface, and the top one is the surface a short time prior the end of the run.

As expected for a pure shadowing MC simulation (Fig. 5a) the roughening due to quasi-linear shadowing is close to the actual observed roughening (Eq. (8)). Nonlinear shadowing is below the actual observed roughening but approaches up to $25 \%$ of the quasi-linear one (as seen in Fig. 5b).

\section{KARDAR-PARISI-ZHANG NONLINEARITY}

The nonlinear generalization of the Kardar-ParisiZhang (KPZ) equation [13] describing the roughening of a surface is given by the evolution of the surface height

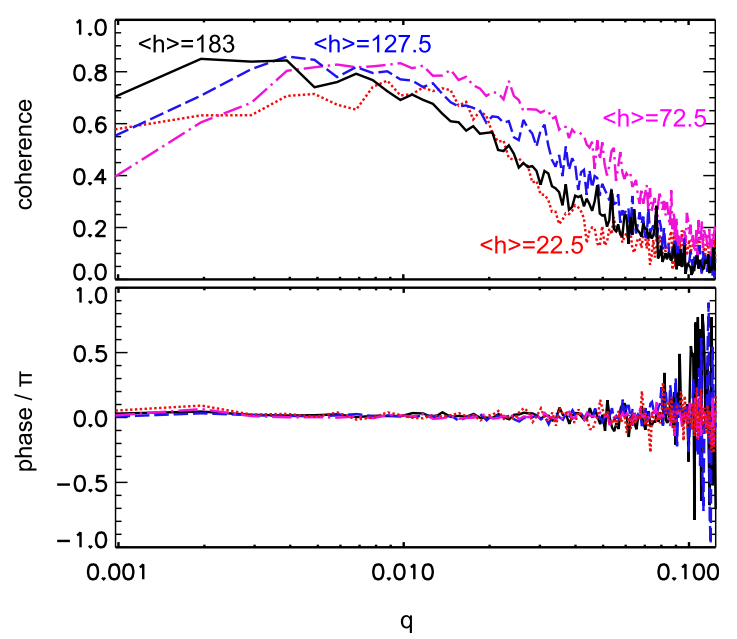

FIG. 7: Cross-coherence and -phase between surface height and exposure angle perturbations from MC simulations.

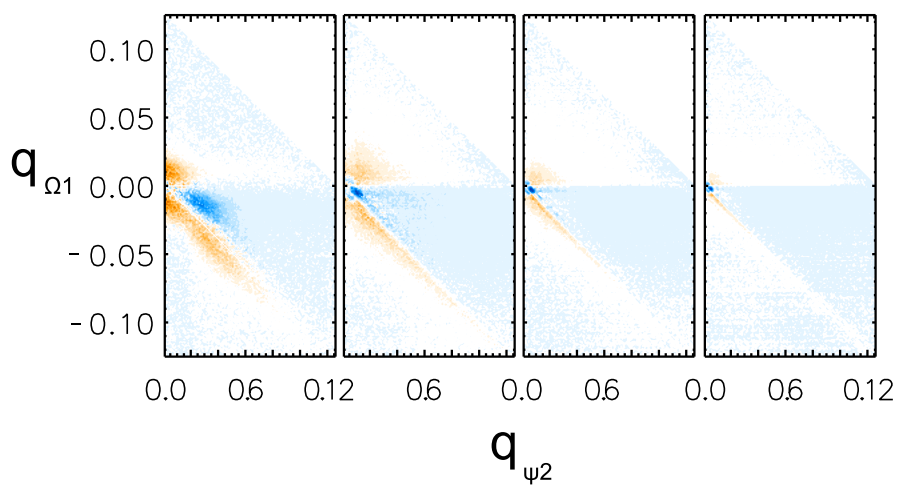

FIG. 8: Evolution of the crossbispectrum between surface height, exposure angle and normal surface growth fluctuations from MC simulations in the same representation as in Fig. 3.

$h(\mathbf{r}, t)$

$$
\frac{\partial h}{\partial t}=\nu \nabla^{2} h+\frac{1}{2} \lambda(\nabla h)^{2}-K \nabla^{4} h+\eta .
$$

Additional to the surface tension $\nu$ the surface selfdiffusion $-K \nabla^{4} h$ give the surface relaxation mechanisms, where $K$ is a temperature dependent positive coefficient. Usually $\nu$ is positive and surface tension dominates over the surface self-diffusion. In this case, the system is morphologically stable. A negative value of $\nu$ implements morphological instability. The balance between only the surface tension (with $\nu<0$ ) and self-diffusion results in periodic height modulation (ripple) with wavelength $\lambda_{B H}=2 \pi \sqrt{2 K /|\nu|}$ called Bradley-Harper instability [14], The term $\frac{1}{2} \lambda(\nabla h)^{2}$ is the lowest order nonlinearity possible and is called KPZ-nonlinearity. Taking $\nu<0$ and also the KPZ-nonlinearity into account Eq. (9) is called the Kuramoto-Sivashinsky equation. In respect to $\nabla h$ the KPZ-nonlinearity acts like self-advection, which can be seen by applying $\nabla$ on Eq. (9) yielding to a 


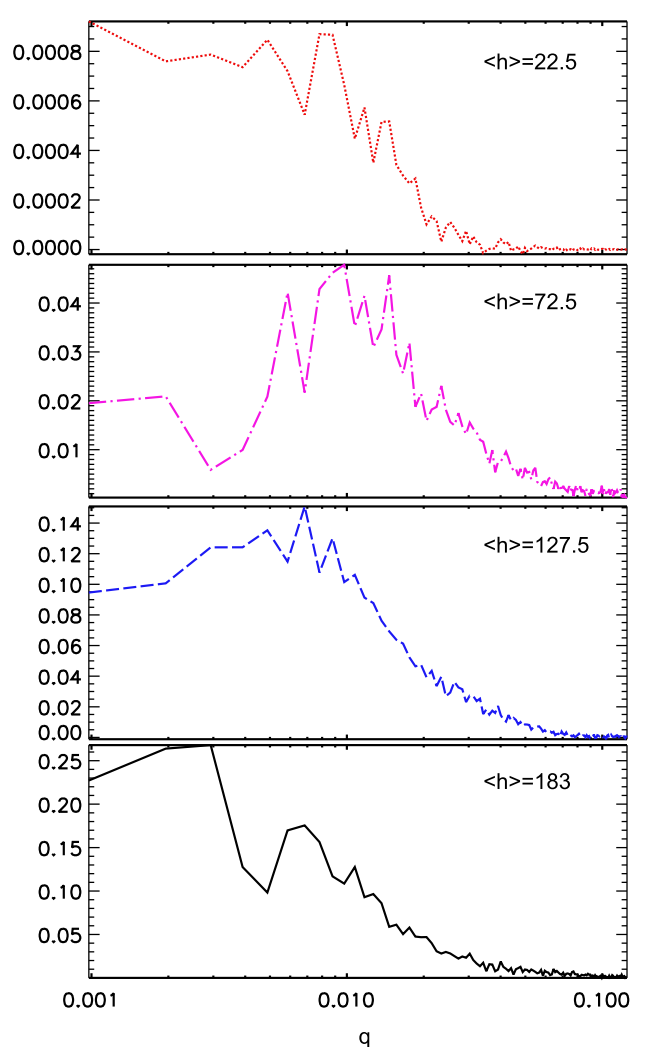

FIG. 9: Integrated crossbispectrum between surface height, exposure angle and normal surface growth fluctuations from MC simulations.

Burgers-like equations, where the velocity is represented by $\nabla h$. A strong self-advection can result in a nonlinear KPZ-instability. For weak self-advection, the linear growth is saturated by the KPZ-nonlinearity, which can result in pattern formation.

In Fourier space, where the surface height is given by $\sum_{\mathbf{q}} h(\mathbf{q}, t) e^{i \mathbf{q r}}$, the KPZ-equation (9) transforms to

$$
\begin{aligned}
& \frac{\partial}{\partial t} \sum_{\mathbf{q}_{1}} h\left(\mathbf{q}_{1}, t\right) e^{i \mathbf{q}_{1} \mathbf{r}}=\sum_{\mathbf{q}_{1}}-\nu q_{1}^{2} h\left(\mathbf{q}_{1}, t\right) e^{i \mathbf{q}_{1} \mathbf{r}} \\
& -\sum_{\mathbf{q}_{1}} \sum_{\mathbf{q}_{2}} \frac{1}{2} \lambda \mathbf{q}_{1} \mathbf{q}_{2} h\left(\mathbf{q}_{1}, t\right) h\left(\mathbf{q}_{2}, t\right) e^{i\left(\mathbf{q}_{1}+\mathbf{q}_{2}\right) \mathbf{r}} \\
& -\sum_{\mathbf{q}_{1}} K q_{1}^{4} h\left(\mathbf{q}_{1}, t\right) e^{i \mathbf{q}_{1} \mathbf{r}}+\mathcal{F}(\eta) .
\end{aligned}
$$

The Fourier transform of the stochastic arrival of the particles is given by $\mathcal{F}(\eta)$, which will be neglected for simplification in the following. By multiplying both sides of this equation by $h^{*}(\mathbf{q}, t) e^{-i \mathbf{q r}}$ and integrating over $d \mathbf{r}$, we can make use of the delta function $\int d \mathbf{r} e^{i\left(\mathbf{q}_{1}-\mathbf{q}\right) \mathbf{r}}=$ $\delta\left(\mathbf{q}_{\mathbf{1}}-\mathbf{q}\right)$ and find

$$
\begin{aligned}
\frac{\partial}{\partial t} h(\mathbf{q}, t)^{2} & =-2\left(\nu q^{2}+K q^{4}\right) h(\mathbf{q}, t)^{2} \\
& -\operatorname{Re} \sum_{\mathbf{q}=\mathbf{q}_{1}+\mathbf{q}_{2}} \lambda \mathbf{q}_{1} \mathbf{q}_{2} h\left(\mathbf{q}_{1}, t\right) h\left(\mathbf{q}_{2}, t\right) h^{*}(\mathbf{q}, t),
\end{aligned}
$$

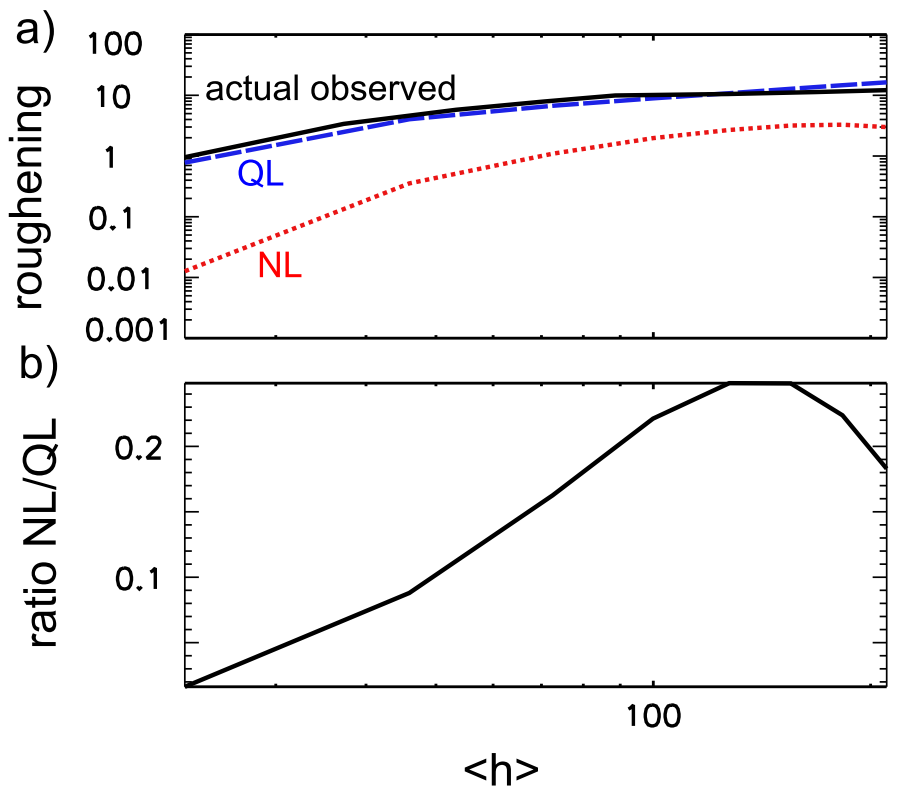

FIG. 10: (a) Comparison of the measured roughening (black, solid) with the roughening due to quasi-linear (QL, blue, dashed) and nonlinear shadowing (NL, red, dotted) for the MC simulation. (b) The ratio between nonlinear and quasilinear roughening.

where the asterisk denotes the complex conjugate. In the case of the KPZ equation, the time evolution of the power spectra $h(\mathbf{q}, t)^{2}$ is determined by linear processes like the surface tension and self-diffusion $\left(-\left(\nu q^{2}+K q^{4}\right)\right)$ and the nonlinear processes, which are reflected by threewave coupling which satisfy the constraint $\mathbf{q}=\mathbf{q}_{\mathbf{1}}+\mathbf{q}_{\mathbf{2}}$.

The basic shape of the KPZ nonlinearity (KPZ-NL) $-q_{1} q_{2} \operatorname{Re}\left\langle h\left(q_{1}\right) h\left(q_{2}\right) h^{*}(q)\right\rangle$ as estimated experimentally is shown in Fig. 11. Before cusps develop (Fig. 11a and b) the nonlinear interactions appear equally distributed over all scales. As cusps arise the KPZ-NL is dominated by local interactions (in $q$-space) at large scales. Stripes develop progressively (Fig. 11e-f), which are nonlocal interactions (in $q$-space) indicating modulational instabilities [15]. As seen in the integrated values (Fig. 12) the KPZNL basically transfers the fluctuations from larger to intermediate wave numbers. As the interactions are mainly local this depicts a forward mass cascade, where largescale cusps break apart in smaller structures. It should be noted that this redistribution is only possible, if the mass input is located at large scales, which is provided by the shadowing instability. Unfortunately the nonlinear coupling coefficient $\lambda$ is not know and the roughening due to the KPZ-NL cannot be quantified. However, to get an impression we set $\lambda=R$ and assuming that the mass at the low $q$ transferred to the intermediate $q$ is filled up by shadowing at low $q$. The corresponding roughening due to the nonlinear redistribution is shown in Fig. 4. The roughening due to the KPZ-NL nearly fits the observed roughening. Therefore the KPZ-NL can 


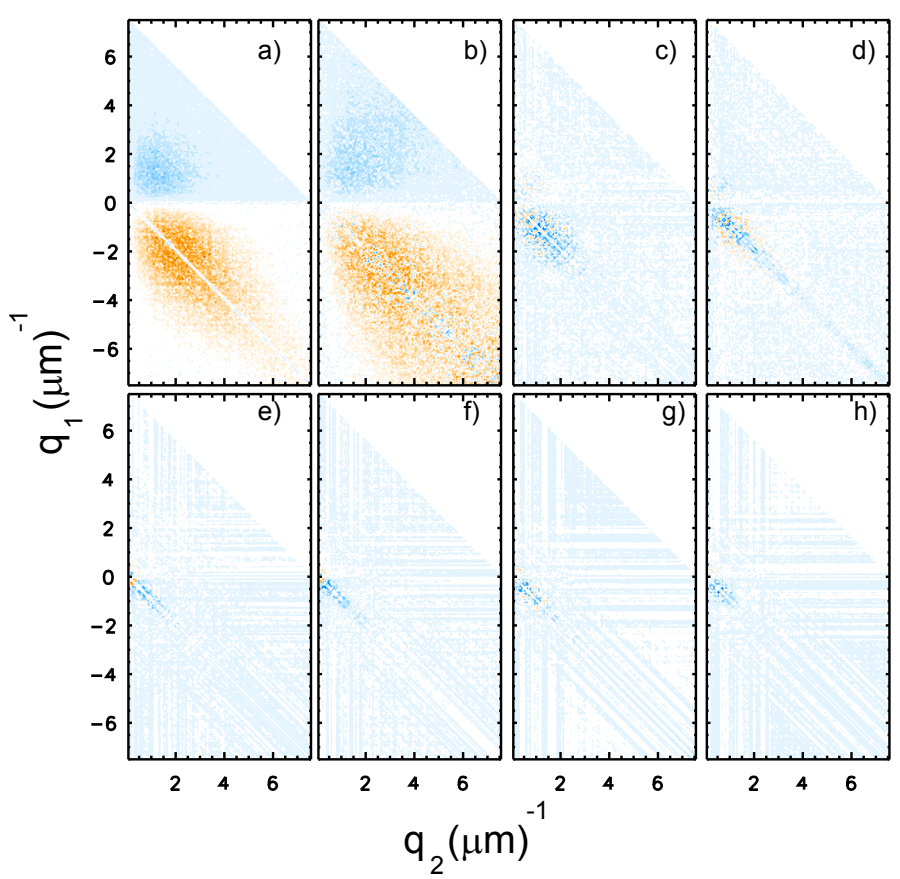

FIG. 11: Bispectrum (weighted by $-q_{1} q_{2}$ ) between surface height fluctuations during fluorocarbon thin-film deposition in the same representation as in Fig. 3.

account for the observed roughening with $\lambda \geq R$.

\section{SUMMARY AND CONCLUSION}

Fluorocarbon thin-film deposition, which sows an anomalous high dynamic scaling exponent of $\beta=1.35$ [4] is investigated in detail. As the shadowing instability reports the highest $\beta$ of close to one, this study is focused on the shadowing instability. Under shadowing larger structures shield the incoming flux to smaller structures and therefore they gain more flux themselves. The shadowing nonlinearity depends mainly on the exposure angle, which is a highly nonlinear function of the surface height. As no general analytical relation between the surface height perturbation and the exposure angle exists the exposure angle is treated as an independent function. Furthermore the investigation is extended to study the influence of normal growth to the surface, which is also a highly nonlinear function of the surface height perturbation and also treated as an independent quantity, here. Surface height perturbations induce perturbations in the exposure angle at the same wavenumber, which are in phase. These modes can couple quasi-linear and result in what we call here quasi-linear shadowing. Furthermore normal growth perturbations are induced at the doubled wavenumber which are expected to be quadrature to the surface height pertubations. Additionally the normal growth modes are modulated by the large-scale surface height structures, which can result in additional

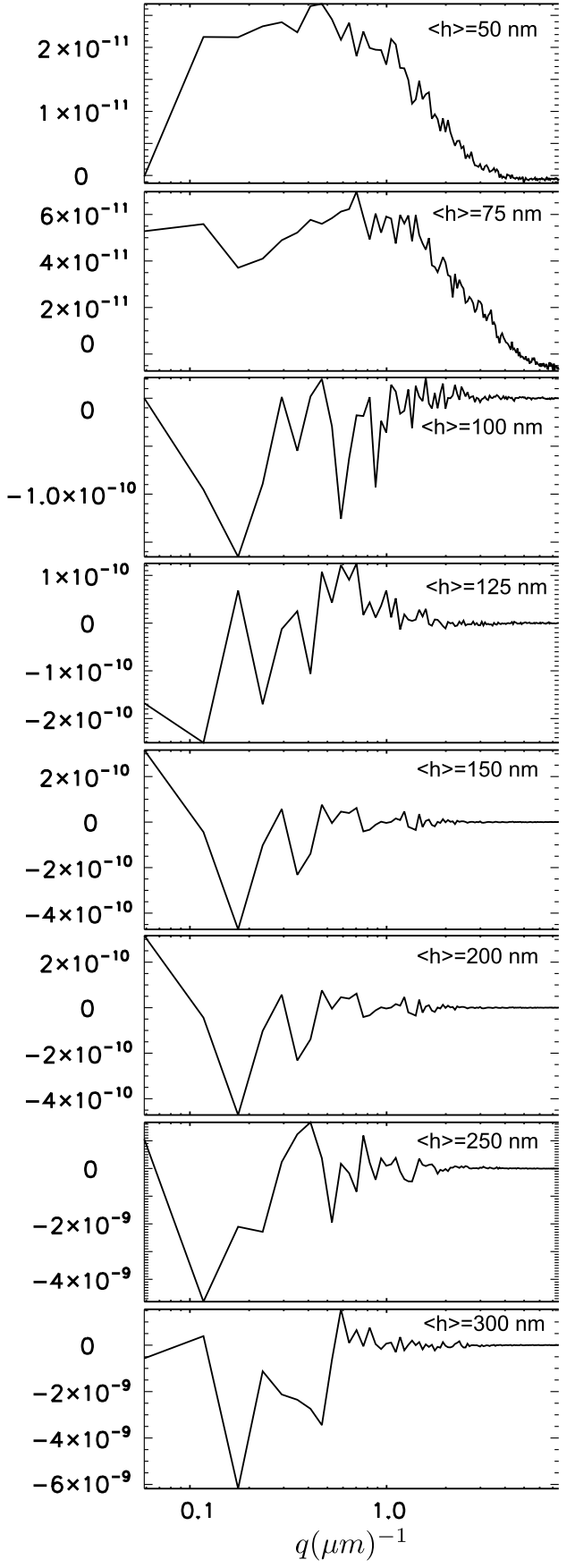

FIG. 12: Integrated bispectrum (weighted by $-q_{1} q_{2}$ ) between surface height fluctuations during fluorocarbon thin-film deposition.

nonlinear modulational roughening. All three mechanisms (quasi-linear and nonlinear modulational roughening as well as nonlinear relaxation due to wavenumber doubling in the normal growth) are present in the experiment. Although the anomalous high dynamical scaling exponent exceeding one cannot be explained by shadowing alone.

The basic considerations are also tested on numerical 
surface morphology data, obtained from a simple MonteCarlo simulation. Quasi-linear roughening is strong enough to explain the observed roughening for the simulation. Here the nonlinear effects show significant contribution to the observed roughening.

As the experiments also report a static scaling exponent of $\alpha=2 / 3$, which is characteristic for nonlinear behavior, also the KPZ nonlinearity is studied. The KPZ nonlinearity does act as a forward cascade under shadowing, transferring fluctuations in the surface height from the scales of the shadowing to intermediate scales, increasing the roughness. The interplay between these two nonlinearities can qualitatively explain a scaling exponent higher than one. The actual roughening due to the KPZ nonlinearity cannot be measured from the experimental data. However, it can be estimated that the nonlinear coupling coefficient of the KPZ nonlinearity has to exceed the linear growth rate to explain the observed anomalous high $\beta$. With in situ real-time measurements of thin film deposition [16] it would be possible to estimate the linear growth rate and the nonlinear coupling coefficients by a spectral power transfer analysis as done in Refs. [17, 18].
[1] A.-L. Barabasi and H. E. Stanley, Fractal Concepts in Surface Growth (University Press, Cambridge, UK, 1995).

[2] R. M. Bradley, Phys. Rev. B 83, 075404 (2011).

[3] G. S. Bales, R. Bruinsma, E. A. Eklund, R. Karunasiri, J. Rudnick, and A. Zangwill, Science 249, 264 (1990).

[4] T. Baloniak and A. von Keudell, Plasma Process. Polym. 5, 653 (2008).

[5] P. A. Premkumar, S. A. Starostin, H. de Vries, M. Creatore, P. M. Koenraad, W. A. MacDonald and M. C. M. van de Sanden, Plasma Process. Polym. 9, 1194 (2012).

[6] M. Drábik, O. Polonskyi, O. Kylián, J. Čechvala, A. Artemenko, I. Gordeev, A. Choukourov, D. Slavinská I. Matolinová and H. Biederman, Plasma Process. Polym. 7, 544 (2010).

[7] G. S. Bales and A. Zangwill, J. Vac. Sci. Technol. A 9, 145 (1991).

[8] R. P. U. Karunasiri, R. Bruinsma, and J. Rudnick, Phys. Rev. Lett. 62, 788 (1989).
[9] T. Karabacak, Y.-P. Zhao, G.-C. Wang, and T.-M. Lu, Phys. Rev. B 64, 085323 (2001).

[10] J.-H. Yao and H. Guo, Phys. Rev. E 47, 1007 (1993).

[11] M. Pelliccione, T. Karabacak, C. Gaire, G.-C. Wang, and T.-M. Lu, Phys. Rev. B 74, 125420 (2006).

[12] Y. K. Kim and E. J. Powers, Phys. Fluids 21, 1452 (1978).

[13] M. Kardar, G. Parisi, and Y.-C. Zhang, Phys. Rev. Lett. 56, 889 (1986).

[14] R. M. Bradley and J. M. E. Harper, J. Vac. Sci. Technol. A 6, 2390 (1988).

[15] P. Manz, M. Ramisch, and U. Stroth, Phys. Rev. Lett. 103, 165004 (2009).

[16] M. J. Rost, Phys. Rev. Lett. 99, 266101 (2007).

[17] P. Manz, M. Ramisch, U. Stroth, V. Naulin, and B. D. Scott, Plasma Phys. \& Controlled Fusion 50, 035008 (2008).

[18] P. Manz, M. Xu, S. C. Thakur, and G. R. Tynan, Plasma Phys. \& Controlled Fusion 53, 095001 (2011). 